\title{
Dementia due to Meningovascular Syphilis in Medial Temporal Lobe and Cognitive Rehabilitation
}

\author{
Seungho Ahn, M.D., Kwang-Ik Jung, M.D., Ph.D., Woo-Kyoung Yoo, M.D., Ph.D., \\ Ga Young Kang, M.D., Suk Hoon Ohn, M.D.
}

Department of Physical Medicine and Rehabilitation, Hallym University College of Medicine, Anyang 431-070, Korea

The temporal lobe is essential in saving declarative memory and plays an important role along with the cerebral neocortex in creating and maintaining long-term memory. Damage to the temporal lobe is expected to result in cognitive impairment or dementia, which has characteristic symptoms such as cognitive and behavioral dysfunction and decreasing self-reliance in activities of daily living. We report on a patient, who suffered from dementia due to meningovascular syphilis affecting the medial temporal lobe, and on the outcome of cognitive rehabilitation.

Key Words Dementia, Meningovascular syphilis, Cognitive rehabilitation

\section{INTRODUCTION}

The medial temporal lobe can be subdivided into the hippocampus, entorhinal cortex, perirhinal cortex, and parahippocampal cortex. It plays a crucial role in the memory system. A form of long term memory, declaratory memory, is concerned with the capacity for conscious recollection of facts and events and is dependent on the structure of the medial temporal

Received June 27, 2011; Accepted September 15, 2011

Corresponding author: Suk Hoon Ohn

Department of Physical Medicine and Rehabilitation, Hallym University College of Medicine, 896, Pyeongchon-dong, Dongan-gu, Anyang 431070, Korea

Tel: +82-31-380-3860, Fax: +82-31-380-3864, E-mail: myeom@korea. com, ohnsh@hallym.ac.kr

(c) This is an open-access article distributed under the terms of the Creative Commons Attribution Non-Commercial License (http:// creativecommons.org/licenses/by-nc/3.0) which permits unrestricted noncommercial use, distribution, and reproduction in any medium, provided the original work is properly cited.

Copyright (C) 2012 by Korean Academy of Rehabilitation Medicine lobe. The hippocampus and parahippocampal cortex are places where new informations are recollected, and the perirhinal cortex is a place which is necessary for familiarity-based recognition. ${ }^{1}$

Lesions involving the medial temporal lobe may therefore be characterized by a severe memory deficit. The authors of this report were presented with a patient suffering from cognitive impairment, depression and decreased activities of daily living arising from meningovascular syphilis affecting the medial temporal lobe.

\section{CASE REPORT}

The subject of this report was a 47-year-old woman, whose academic ability was at the level of a high school graduate. She had suffered from a gradual deterioration of gait and memory for the past three years. Two years ago, she was diagnosed with a cervical intervertebral 
disc herniation for her gait disturbance and underwent cervical disc surgery. During the preparations for her cervical disc operation, she was diagnosed by a serological test as suffering from syphilis and turned out to have neurosyphilis on cerebrospinal fluid analysis. Nine months after the operation, she was readmitted for the treatment of her neurosyphilis. Magnetic resonance imaging (MRI) of her brain revealed lesions in the bilateral medial temporal lobes, insula and left cerebellar hemisphere (Fig. 1-A). The lesions in the bilateral medial temporal lobes and insula proved to be the result of neurosyphilis, and those in the left cerebellum proved to be a meningioma.

To investigate the cognitive impairment, the patient was assessed using the ' 30 -minute neuropsychological assessment protocol developed by the National Institute of Neurological Disorders and Stroke and the Canadian Stroke Network in 2006. ${ }^{2}$ The '30-minute neuropsychological assessment protocol includes nine tests: 1) animal naming, 2) the Controlled Oral Word Association Test (COWAT), 3) the digit symbol coding subtest of the Wechsler Adult Intelligence Scale, 3rd edition, 4) the Hopkins Verbal Learning Test-Revised Version (HVLT-R), 5) the Center for Epidemiologic Studies-Depression Scale (CES-D), 6) the Neuropsychiatric Inventory-Questionnaire Version (NPI-Q), 7) the Mini-Mental State Examination (MMSE), 8) the Trail Making Test-Part A, and 9) the Trail Making Test-Part B. Korean versions were used for the COWAT, HVLT-R, and MMSE. While the original version of the COWAT includes two sets of three letters (CFL or PRW), the Korean version (K-COWAT) includes three Korean consonants: ᄀ, O , 入. Additional tests used were the digit span test (forward and backward), the Rey-Osterreith Complex Figure Test (RCFT), the Global Deterioration Scale (GDS), and the Clinical Dementia Rating (CDR) scale. The detailed results of the cognitive function tests are shown in Table 1. Problems were found in the domains of attention, the registration and saving of information, and perception.

After two weeks of treatment with penicillin, the patient had surgery for the removal of the cerebellar tumor. After the surgery, a cerebral infarction and hemorrhagic transformation occurred in the left temporal lobe and inferior frontal gyrus (Fig. 1-B). After the stroke, the patient's cognitive function deteriorated, and after one year she visited a department of neurorehabilitation because of cognitive impairment and aphasia. Cognitive function was re-assessed with the same protocol as before, but with the addition of a language function test, the Korean version of the Western Aphasia Battery (K-WAB). The results of the cognitive function tests one year after the stroke are included in Table 1 and clearly confirm that a general deterioration in the patient's cognitive function had occurred. Moreover, the patient was diagnosed with conduction aphasia on the basis of the $\mathrm{K}-\mathrm{WAB}$ results.

Cognitive neurorehabilitation was initiated and continued for six weeks. This involved attention training, verbal and visuospatial memory training, perception
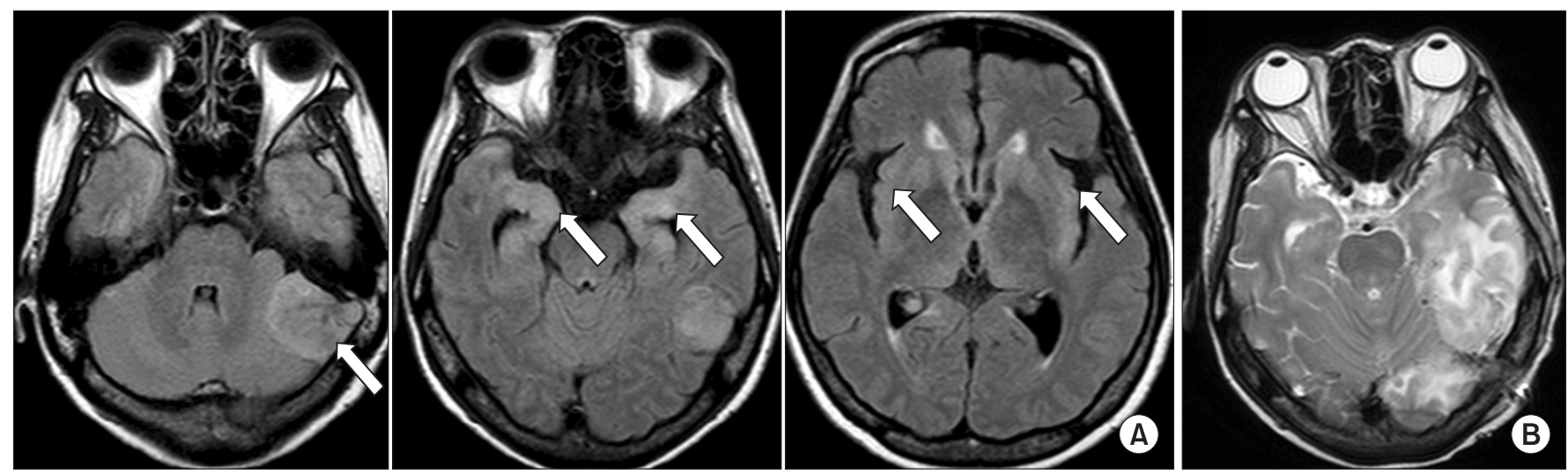

Fig. 1. MRI performed before and after cerebellar meningioma removal operation. (A) T1 MRI that shows signal change noted in both medial temporal lobe and left cerebellar hemisphere. The lesions in the bilateral medial temporal lobe are neurosyphilis, and the lesion in the left cerebellar hemisphere is a meningioma (white arrows). (B) T2 MRI that shows cerebral infarction with hemorrhagic transformation in left temporal lobe. 
Table 1. Test Results following Presentation with Neurosyphilis and after Stroke and Cognitive Rehabilitation

\begin{tabular}{|c|c|c|c|c|}
\hline & $\begin{array}{c}\text { Neuropsychological } \\
\text { test }\end{array}$ & After neurosyphilis & After stroke & $\begin{array}{l}\text { After rehabilitation } \\
\text { for } 6 \text { weeks }\end{array}$ \\
\hline Animal Naming & & 10 & 6 & 9 \\
\hline \multirow[t]{3}{*}{ K-COWAT } & $\neg$ & 1 & 1 & 5 \\
\hline & o & 3 & 2 & 3 \\
\hline & 入 & 3 & 1 & 1 \\
\hline Digit symbol coding & & Correct 23 & Correct 10 & Correct 15 \\
\hline \multirow[t]{5}{*}{ K-HVLT } & Free recall-1st & 1 & 1 & 1 \\
\hline & Free recall-2nd & 7 & 1 & 1 \\
\hline & Free recall-3rd & 7 & 2 & 2 \\
\hline & 20 min delayed recall & 5 & 0 & 1 \\
\hline & Recognition & $47.7 \%$ & $0 \%$ & $23.0 \%$ \\
\hline CES-D & & 20 & 20 & 9 \\
\hline NPI-Q & & 5 & 5 & 3 \\
\hline MMSE-K & & 18 & 15 & 20 \\
\hline Trail making-A & & $1 \mathrm{~min} 14 \mathrm{sec}$ & Fail & $2 \min 20 \mathrm{sec}$ \\
\hline Trail making-B & & Fail & Fail & Fail \\
\hline \multirow[t]{2}{*}{ Digit span } & Forward & 4 & 2 & 3 \\
\hline & Backward & 3 & 0 & 2 \\
\hline \multirow[t]{3}{*}{ RCFT } & Copy & 23 & Fail & 24 \\
\hline & Immediate recall & 7.5 & Fail & 3 \\
\hline & Delayed recall & 3 & Fail & Fail \\
\hline GDS & & 4 & 4 & 3 \\
\hline CDR & & 1 & 2 & 0.5 \\
\hline
\end{tabular}

K-COWAT: Korean Controlled oral word association test, K-HVLT: Korean Hopkins verbal learning test, CES-D: Center for epidemiological studies-depression scale, NPI-Q: Neuropsychiatric inventory questionnaire, MMSE-K: MiniMental state examination-Korean version, RCFT: Rey complex figure test, GDS: Global deterioration scale, CDR: Clinical dementia rating

training and executive training for one hour six days a week. The patient was also treated with donepezil $5 \mathrm{mg}$ and methylphenidate $10 \mathrm{mg}$ during that time. After the 6 -week treatment, cognitive function was further reassessed (Table 1), and the results at this stage revealed improvements in semantic fluency, phonemic fluency, working memory, attention, recognition, and perception.

\section{DISCUSSION}

This report illustrates the case of a patient, who had bilateral temporal lesions and severe problems in almost all cognitive domains, secondary to neurosyphilis and following a cerebrovascular accident. The patient's dementia resulted from medial temporal lobe dysfunction. Medial temporal lobes compose a memory network, which includes the anterior thalamic nucleus, mammillary body, fornix, and prefrontal cortex. ${ }^{3}$ The medial temporal lobes have a direct corticocortical connection with the prefrontal cortex, passing through the uncinate fascicle, anterior temporal stem and anterior corpus callosum. Orbitofrontal and dorsolateral prefrontal cortices have a strong connection with perirhinal and entorhinal cortices, and the ventromedial prefrontal cortex has a strong connection with the hippocampus CAl region. In addition, the medial temporal lobes receive information from sensory association areas. Information originating from extensive cerebral areas enters predominantly through the perirhinal and parahippocampal cortices and then projects to the entorhinal cortex and hippocampus. Information is stored as long term memory as the result of repetitive 
retrieval and rehearsal. ${ }^{3}$ Based on these broad networks, the temporal lobes are essential for the storage of declarative memory and play an important role along with cerebral neocortex in creating and maintaining long-term memory. ${ }^{4}$ Patients with lesions in the medial temporal lobe have shown impairments in various cognitive domains.,

Neurosyphilis refers to an infection of the brain and spinal cord caused by Treponema pallidum. Neurosyphilis has a diverse clinical presentation. The major clinical forms of neurosyphilis are syphilitic meningitis, meningovascular syphilis, parenchymatous syphilis, and gummatous syphilis. Meningovascular syphilis refers to the arteritis and meningitis caused by neurosyphilis and may result in cerebrovascular accidents. ${ }^{7}$ In this case, the cerebral infarction in the left medial temporal lobe occurred after surgical removal of the cerebellar tumor, and we were therefore unable to completely rule out the possibility of a direct operative effect as a cause of the infarction. We discussed this case with a neurosurgeon and an anesthesiologist, and we concluded that the possibility of direct operative trauma was very low, because the operation was performed in the infratentorium, and infarction occurred in the supratentorium. The possibility of infarction due to hypovolemia was also very low, because volume was controlled strictly throughout the surgical procedure.

This patient suffered from conduction aphasia after the cerebral infarction. In terms of a language processing network, it is thought that dorsal and ventral pathways form a concurrent parallel system which connects sensory and motor language areas. ${ }^{8}$ In Saur's study, the dorsal pathway connecting superior temporal regions and premotor regions along the arcuate fasciculus and superior longitudinal fasciculus was activated during repetition. In contrast, the ventral pathway connecting the middle/inferior temporal regions and the ventrolateral prefrontal cortex was activated during auditory comprehension. Since the patient demonstrated particular impairments in repetition and naming, we could therefore assume that the dorsal pathway was more severely impaired than the ventral pathway.

After neurorehabilitation for six weeks, improvements had occurred in mood and in many cognitive domains, including fluency, recognition, spatial and verbal attention. Marked improvements were found on the
MMSE-K, K-COWAT, and RCFT. Since neurorehabilitation including medication and conventional cognitive retraining was started in the chronic stage of post-stroke dementia, we suggest that the cognitive improvements were due to neurorehabilitation. However, information saving based on the total and delayed recall scores did not improve, even though recognition improvement was preserved. Ranjith et al. reported that a frontotemporal dementia group made fewer errors on a recognition task than Alzheimer dementia and vascular dementia groups. ${ }^{9}$ Our report also suggests that patients with dementia due to temporal lesions do not completely lose already encoded information.

There are two major limitations in this case report. Firstly, we did not use functional neuroimaging to support the behavioral data. The second limitation is that the patient had a brain tumor in her left cerebellum. After the tumor was removed, mild post-operative ischemia or hemorrhage was inevitable. We should therefore not overlook possible contribution of cerebellar lesions to the patient's cognitive impairments. Recent clinical studies have shown that the cerebellum is also a part of the cognitive network. ${ }^{10}$ The patient's deteriorated cognitive function after the surgery can be explained not only by the infarction in the medial temporal lobe but also by a cerebellar lesion. However, if we compare the usual characteristics of cognitive dysfunctions due to lesions in the medial temporal lobe and the cerebellum, the patient's dementia symptoms reflected dysfunctions in the medial temporal lobe rather than in the cerebellum because the symptoms appeared in such a broad spectrum of cognitive domains.

In summary, we have described the presentation and treatment of a patient suffering from dementia due to meningovascular syphilis in the medial temporal lobes, and we were able to confirm that the dementia symptoms could be improved by short-term comprehensive neurorehabilitation involving medication and daily sessions of cognitive training.

\section{REFERENCES}

1. Snaphaan L, Rijpkema M, van Uden I, Fernandez G, de Leeuw FE. Reduced medial temporal lobe functionality in stroke patients: a functional magnetic resonance imaging study. Brain 2009; 132: 1882-1888 
2. Hachinski V, Iadecola C, Petersen RC, Breteler MM, Nyenhuis DL, Black SE, Powers WJ, DeCarli C, Merino JG, Kalaria RN, et al. National Institute of Neurological Disorders and Stroke-Canadian Stroke Network vascular cognitive impairment harmonization standards. Stroke 2006; 37: 2220-2241

3. Daselaar SM, Veltman DJ, Witter MP. Common pathway in the medial temporal lobe for storage and recovery of words as revealed by event-related functional MRI. Hippocampus 2004; 14: 163-169

4. Hales JB, Brewer JB. Activity in the hippocampus and neocortical working memory regions predicts successful associative memory for temporally discontiguous events. Neuropsychologia 2010; 48: 33513359

5. Jenkins LJ, Ranganath C. Prefrontal and medial temporal lobe activity at encoding predicts temporal context memory. J Neurosci 2010; 30: 15558-15565

6. McKinnon MC, Nica EI, Sengdy P, Kovacevic N, Moscovitch M, Freedman M, Miller BL, Black SE,
Levine B. Autobiographical memory and patterns of brain atrophy in frontotemporal lobar degeneration. J Cogn Neurosci 2008; 20: 1839-1853

7. Asdaghi N, Muayqil T, Scozzafava J, Jassal R, Saqqur $\mathrm{M}$, Jeerakathil TJ. The re-emergence in Canada of meningovascular syphilis: 2 patients with headache and stroke. CMAJ 2007; 176: 1699-1700

8. Saur D, Kreher BW, Schnell S, Kümmerer D, Kellmeyer P, Vry MS, Umarova R, Musso M, Glauche V, Abel S, et al. Ventral and dorsal pathways for language. Proc Natl Acad Sci U S A 2008; 105: 18035-18040

9. Ranjith N, Mathuranath PS, Sharma G, Alexander A. Qualitative aspects of learning, recall, and recognition in dementia. Ann Indian Acad Neurol 2010; 13: 117122

10. Baillieux H, De Smet HJ, Paquier PF, De Deyn PP, Marien P. Cerebellar neurocognition: insights into the bottom of the brain. Clin Neurol Neurosurg 2008; 110: 763-773 